\title{
n-Refined Neutrosophic Vector Spaces
}

\author{
Florentin Smarandache ${ }^{1}$ and Mohammad Abobala ${ }^{2}$ \\ 1 Department of Mathematics and Science, University of New Mexico, Gallup, NM 87301, USA \\ ${ }^{2}$ Tishreen University, Faculty of Science, Department of Mathematics, Lattakia, Syria \\ ${ }^{1}$ e-mail: smarand@unm.edu \\ 2.e-mail: mohammadabobala777@gmail.com
}

\begin{abstract}
This paper introduces the concept of n-refined neutrosophic vector spaces as a generalization of neutrosophic vector spaces, and it studies elementary properties of them. Also, this work discusses some corresponding concepts such as weak/strong n-refined neutrosophic vector spaces, and n-refined neutrosophic homomorphisms.
\end{abstract}

Keywords: n-Refined weak neutrosophic vector space, n-Refined strong neutrosophic vector space, n-Refined neutrosophic homomorphism.

\section{Introduction}

Neutrosophy as a part of philosophy founded by F. Smarandache to study origin, nature, and indeterminacies became a strong tool in studying algebraic concepts. Neutrosophic algebraic structures were defined and studied such as neutrosophic modules, and neutrosopohic vector spaces, etc.See $[1,2,3,4,5,6,7,8,9]$. In 2013 Smarandacheintroduceda perfect idea, when he extended the neutrosophic set to refined [n-valued] neutrosophic set, i.e. the truth value $T$ is refined/split into types of sub-truths such as $\left(T_{1}, T_{2}, \ldots,\right)$ similarly indeterminacy $I$ is refined/split into types of sub-indeterminacies $\left(\mathrm{I}_{1}, \mathrm{I}_{2}, \ldots,\right)$ and the falsehood $\mathrm{F}$ is refined/split into sub-falsehood ( $\mathrm{F}_{1}$, $\left.F_{2}, . .,\right)[10,11]$. Refined neutrosophic algebraic structures were studied such as refined neutrosophic rings, refined neutrosophic modules, and n-refined neutrosophic rings [4,12].

In this article authors try to define n-refined neutrosophic vector spaces, subspaces, and homomorphisms and to present some of their elementary properties.

For our purpose we use multiplication operation (defined in [12]) between indeterminacies $I_{1}, I_{2}, \ldots, I_{n}$ as follows:

$$
I_{m} I_{s}=I_{\min (m, s)}
$$

This work is a continuation of the study on the n-refined neutrosophic structures that began in [12].

\section{Preliminaries}


Definition 2.1: [12]

Let $(\mathrm{R},+, \mathrm{x})$ be a ring and $I_{k} ; 1 \leq k \leq n$ be $\mathrm{n}$ indeterminacies. We define $R_{n}(\mathrm{I})=\left\{a_{0}+a_{1} I+\cdots+a_{n} I_{n} ; a_{i} \in R\right\}$ to be an n-refined neutrosophic ring.

Definition 4.3: [12]

(a) Let $R_{n}$ (I) be an n-refined neutrosophic ring and $\mathrm{P}=\sum_{i=0}^{n} P_{i} I_{i}=\left\{a_{0}+a_{1} I+\cdots+a_{n} I_{n}: a_{i} \in P_{i}\right\}$, where $P_{i}$ is a subset of R, we define $\mathrm{P}$ to be an AH-subring if $P_{i}$ is a subring of $\mathrm{R}$ for all. AHS-subring is defined by the condition $P_{i}=P_{j}$ for all $i, j$.

(b) $\mathrm{P}$ is an AH-ideal if $P_{i}$ aretwo-side ideals of $\mathrm{R}$ for all $i$, the AHS-ideal is defined by the condition $P_{i}=P_{j}$ for all $i, j$.

(c) The AH-ideal $\mathrm{P}$ is said to be null if $P_{i}=\operatorname{Ror} P_{i}=\{0\}$ for alli.

Definition $2.3:[5]$

Let $(\mathrm{V},+, \cdot)$ be a vector space over the field $\mathrm{K}$; then $(\mathrm{V}(\mathrm{I}),+, \cdot)$ is called a weak neutrosophic vector space over the field $\mathrm{K}$, and it is called a strong neutrosophic vector space if it is a vector space over the neutrosophic field $\mathrm{K}(\mathrm{I})$.

Definition $2.4:[5]$

Let V(I) be a strong neutrosophic vector space over the neutrosophic field $\mathrm{K}(\mathrm{I})$ and $\mathrm{W}(\mathrm{I})$ be a non empty set of V(I) then W(I) is called a strong neutrosophic subspace if W(I) is itself a strong neutrosophic vector space.

Definition $2.6:[5]$

Let $\mathrm{U}(\mathrm{I}), \mathrm{W}(\mathrm{I})$ be twostrong neutrosophic subspaces of $\mathrm{V}(\mathrm{I})$ and let $f: V(I) \rightarrow W(I)$, we say that $\mathrm{f}$ is a neutrosophic vector space homomorphism if

(a) $f(I)=I$,

(b) $\mathrm{f}$ is a vector space homomorphism.

We define the kernel of $\mathrm{f}$ by $\operatorname{Ker}(\mathrm{f})=\left\{\mathrm{x} \in V(I) ; \mathrm{f}(\mathrm{x})=0_{W(I)}\right\}$.

Definition $2.7:[5]$

Let $v_{1}, v_{2} . . v_{s} \in V(I)$ and $x \in V(I)$; we say that $\mathrm{x}$ is a linear combination of $\left\{v_{i} ; i=1, \ldots, s\right\}$ if

$\mathrm{x}=a_{1} v_{1}+\cdots+a_{s} v_{s}$ suchthat $a_{i} \in K(I)$.

The $\operatorname{set}\left\{\boldsymbol{v}_{\boldsymbol{i}} ; \boldsymbol{i}=\mathbf{1}, \ldots, \boldsymbol{s}\right\}$ is called linearly independent if $\boldsymbol{a}_{\mathbf{1}} \boldsymbol{v}_{\mathbf{1}}+\cdots+\boldsymbol{a}_{\boldsymbol{s}} \boldsymbol{v}_{\boldsymbol{s}}=\mathbf{0}$ implies $\boldsymbol{a}_{\boldsymbol{i}}=\mathbf{0}$ for all i.

\section{Main concepts and results}

Definition 3.1:

Let $(\mathrm{K},+, \cdot)$ be a field, we say that $K_{n}(I)=K+K I_{1}+\cdots+K I_{n}=\left\{a_{0}+a_{1} I_{1}+\cdots+a_{n} I_{n} ; a_{i} \in K\right\}$ is an n-refined neutrosophic field.

It is clear that $K_{n}(I)$ is an n-refined neutrosophic field, but not a field in the classical meaning. 


\section{Example 3.2 :}

Let $K=Q$ be the field of rationals. The corresponding 3-refined neutrosophic field is

$$
Q_{3}(I)=\left\{a+b I_{1}+c I_{2}+d I_{3} ; a, b, c, d \in Q\right\} .
$$

Definition 3.3 :

Let $(\mathrm{V},+, \cdot)$ be a vector space over the field $\mathrm{K}$. Then we say that $V_{n}(I)=V+V I_{1}+\cdots+V I_{n}=\left\{x_{0}+x_{1} I_{1}+\cdots+\right.$ $\left.x_{n} I_{n} ; x_{i} \in V\right\}$ is a weak n-refined neutrosophic vector space over the field K. Elements of $V_{n}(I)$ are called n-refined neutrosophic vectors, elements of $\mathrm{K}$ are called scalars.

If we take scalars from the n-refined neutrosophicfield $K_{n}(I)$, we say that $V_{n}(I)$ is a strong n-refined neutrosophic vector space over the n-refined neutrosophic field $K_{n}(I)$. Elements of $K_{n}(I)$ are called n-refined neutrosophic scalars.

\section{Remark 3.4:}

If we take $n=1$ we get the classical neutrosophic vector space.

Addition on $V_{n}(I)$ is defined as:

$$
\sum_{i=0}^{n} a_{i} I_{i}+\sum_{i=0}^{n} b_{i} I_{i}=\sum_{i=0}^{n}\left(a_{i}+b_{i}\right) I_{i}
$$

Multiplication by a scalar $m \in K$ is defined as:

$$
m \cdot \sum_{i=0}^{n} a_{i} I_{i}=\sum_{i=0}^{n}\left(m . a_{i}\right) I_{i} .
$$

Multiplication by an n-refined neutrosophic scalar $m=\sum_{i=0}^{n} m_{i} I_{i} \in K_{n}(I)$ is defined as:

$$
\left(\sum_{i=0}^{n} m_{i} I_{i}\right) \cdot\left(\sum_{i=0}^{n} a_{i} I_{i}\right)=\sum_{i, j=0}^{n}\left(m_{i} \cdot a_{j}\right) I_{i} I_{j}
$$

where $a_{i} \in V, m_{i} \in K, I_{i} I_{j}=I_{\min (i, j)}$.

Theorem 3.5 :

Let $(\mathrm{V},+, \cdot)$ be a vector space over the field $\mathrm{K}$. Then a weak n-refined neutrosophic vector space $V_{n}(I)$ is a vector space over the field $\mathrm{K}$. A strong $\mathrm{n}$-refined neutrosophic vector space is not a vector space but a module over the nrefinedneutrosophic field $K_{n}(\mathrm{I})$.

Proof:

It is similar to that of Theorem 2.3 in [5].

Example 3.6:

Let $V=Z_{2}$ be the finite vector space of integers modulo 2 over itself:

(a) The corresponding weak 2-refined neutrosophic vector space over the field $Z_{2}$ is

$V_{n}(I)=\left\{0,1, I_{1}, I_{2}, I_{1}+I_{2}, 1+I_{1}+I_{2}, 1+I_{1}, 1+I_{2}\right\}$ 


\section{Definition 3.7:}

Let $V_{n}(I)$ be a weak n-refined neutrosophic vector space over the field $\mathrm{K}$; a nonempty subset $W_{n}(I)$ is called a weak n-refined neutrosophic subspace of $V_{n}(I)$ if $W_{n}(I)$ is a subspace of $V_{n}(I)$ itself.

Definition 3.8:

Let $V_{n}(I)$ be a strong n-refined neutrosophic vector space over the n-refined neutrosophic field $K_{n}(I)$; a nonempty subset $W_{n}(I)$ is called a strong n-refined neutrosophic subspace of $V_{n}(I)$ if $W_{n}(I)$ is a submodule of $V_{n}(I)$ itself.

Theorem 3.9:

Let $V_{n}(I)$ be a weak n-refined neutrosophic vector space over the field $\mathrm{K}, W_{n}(I)$ be a nonempty subset of $V_{n}(I)$. Then $W_{n}(I)$ is a weak n-refined neutrosophic subspace if and only if:

$x+y \in W_{n}(I), m \cdot x \in W_{n}(I)$ for all $x, y \in W_{n}(I), m \in K$.

Proof:

It holds directly from the condition of subspace.

Theorem 3.10:

Let $V_{n}(I)$ be a strong n-refined neutrosophic vector space over an n-refined neutrosophic field $K_{n}(I), W_{n}(I)$ be a nonempty subset of $V_{n}(I)$. Then $W_{n}(I)$ is a strong n-refined neutrosophic subspace if and only if:

$x+y \in W_{n}(I), m \cdot x \in W_{n}(I)$ for all $x, y \in W_{n}(I), m \in K_{n}(I)$.

Proof:

It holds directly from the condition of submodule.

Example 3.11:

Let $V=R^{2}$ be a vector space over the field $\mathrm{R}, W=<(0,1)>$ is a subspace of $\mathrm{V}, R_{2}^{2}(I)=\left\{(a, b)+(m, s) I_{1}+\right.$ $\left.(k, t) I_{2} ; a, b, m, s, k, t \in R\right\}$ is the corresponding weak/strong 2-refined neutrosophic vector space.

$W_{2}(I)=\left\{a_{0}+a_{1} I_{1}+a_{2} I_{2}\right\}=\left\{(0, x)+(0, y) I_{1}+(0, z) I_{2} ; x, y, z \in R\right\}$ is a weak 2-refined neutrosophic subspace of the weak 2-refined neutrosophic vector space $R_{2}^{2}(I)$ over the field R.

$W_{2}(I)=\left\{a_{0}+a_{1} I_{1}+a_{2} I_{2}\right\}=\left\{(0, x)+(0, y) I_{1}+(0, z) I_{2} ; x, y, z \in R\right\}$ is a strong 2-refined neutrosophic subspace of the strong 2-refined neutrosophic vector space $R_{2}^{2}(I)$ over the n-refined neutrosophic field $R_{2}(I)$.

Definition 3.12:

Let $V_{n}(I)$ be a weak n-refined neutrosophic vector space over the field $\mathrm{K}, x$ be an arbitrary element of $V_{n}(I)$, we say that $\mathrm{x}$ is a linear combination of $\left\{x_{1}, x_{2}, \ldots, x_{m}\right\} \subseteq V_{n}(I)$, or $x=a_{1} x_{1}+a_{2} x_{2}+\cdots+a_{m} x_{m}: a_{i} \in K, x_{i} \in V_{n}(I)$.

Example 3.13:

Consider the weak 2-refined neutrosophic vector space in Example 3.11, 
$x=(0,2)+(1,3) I \in R_{2}^{2}(I), \quad x=2(0,1)+1(1,0) I_{1}+3(0,1) I_{2}$, i.e $x$ is a linear combination of the set $\left\{(0,1),(1,0) I_{1},(0,1) I_{2}\right\}$ over the field $\mathrm{R}$.

Definition 3.14:

Let $V_{n}(I)$ be a strong n-refined neutrosophic vector space over an n-refined neutrosophic field $K_{n}(I), x$ be an arbitrary element of $V_{n}(I)$, we say that $\mathrm{x}$ is a linear combination of $\left\{x_{1}, x_{2}, \ldots, x_{m}\right\} \subseteq V_{n}(I)$ is $x=a_{1} x_{1}+a_{2} x_{2}+$ $\cdots+a_{m} x_{m}: a_{i} \in K_{n}(I), x_{i} \in V_{n}(I)$.

Example 3.15:

Consider the strong 2-refined neutrosophic vector space $R_{2}^{2}(I)=\left\{(a, b)+(m, s) I_{1}+(k, t) I_{2} ; a, b, m, s, k, t \in R\right\}$ over the 2-refined neutrosophic field $R_{2}(I)$,

$x=(0,2)+(3,3) I_{1}+(-1,0) I_{2}=\left(2+I_{1}\right) \cdot(0,1)+\left(1+I_{2}\right) \cdot(1,1) I_{1}+\left(I_{1}-I_{2}\right) \cdot(1,0) I_{2}$, hence $\mathrm{x}$ is a linear combination of the set $\left\{(0,1),(1,1) I_{1},(1,0) I_{2}\right\}$ over the 2-refined neutrosophic field $R_{2}(I)$.

Definition 3.16:

Let $X=\left\{x_{1}, \ldots, x_{m}\right\}$ be a subset of a weak n-refined neutrosophic vector space $V_{n}(\mathrm{I})$ over the field $\mathrm{K}, \mathrm{X}$ is a weak linearly independent set if $\sum_{i=0}^{m} a_{i} x_{i}=0$ implies $_{i}=0 ; a_{i} \in K$.

Definition 3.17:

Let $X=\left\{x_{1}, \ldots, x_{m}\right\}$ be a subset of a strong n-refined neutrosophic vector space $V_{n}(\mathrm{I})$ over the n-refined neutrosophic field $K_{n}(I), \mathrm{X}$ is a weak linearly independent set if $\sum_{i=0}^{m} a_{i} x_{i}=0$ impliesa $_{i}=0 ; a_{i} \in K_{n}(I)$.

Definition 3.18:

Let $V_{n}(I), W_{n}(I)$ be two strong n-refined neutrosophic vector space over the n-refined neutrosophic field $K_{n}(I)$, let $f: V_{n}(I) \rightarrow U_{n}(I)$ be a well defined map. It is called a strong n-refined neutrosophic homomorphism if:

$f(a \cdot x+b \cdot y)=a \cdot f(x)+b \cdot f(y)$ for all $x, y \in V_{n}(I), a, b \in K_{n}(I)$.

A weak n-refined neutrosophic homomorphism can be defined as the same.

We can understand the strong n-refined homomorphism as a module homomorphism, weak n-refined neutrosophic homomorphism can be understood as a vector space homomorphism.

Remark:

The previous definition of n-refined homomorphism between two strong/weak n-refined vector spaces is a classical homomorphism between two modules/spaces. We can not add a similar condition to the concept of neutrosophic homomorphism $\left(f\left(I_{i}\right)=I_{i}\right)$, since $I_{i}$ is not supposed to be an element of $V_{n}(I)$ if $V$ has more than one dimension for example. According to our definition, $\operatorname{Ker}(f)$ will be a subspace (which is different from classical neutrosophic vector space case) sicne $f$ was defined as a classical homomorphism without any additional condition.

Definition 3.19:

Let $f: V_{n}(I) \rightarrow U_{n}(I)$ be a weak/strong n-refined neutrosophic homomorphism, we define:

(a) $\operatorname{Ker}(f)=\left\{x \in V_{n}(I) ; f(x)=0\right\}$. 
(b) $\operatorname{Im}(f)=\left\{y \in U_{n}(I) ; \exists x \in V_{n}(I)\right.$ and $\left.y=f(x)\right\}$.

Theorem 3.20:

Let $f: V_{n}(I) \rightarrow U_{n}(I)$ be a weak n-refined neutrosophic homomorphism. Then

(a) $\operatorname{Ker}(f)$ is a weak n-refined neutrosophic subspace of $V_{n}(\mathrm{I})$.

(b) $\operatorname{Im}(f)$ is a weak n-refined neutrosophic subspace of $U_{n}(I)$.

Proof:

(a) $f$ is a vector space homomorphism since $V_{n}(I), U_{n}(I)$ are vector spaces, hence $\operatorname{Ker}(f)$ is a subspace of the vector space $V_{n}(I)$, thus $\operatorname{Ker}(f)$ is a weak n-refined neutrosophic subspace of $V_{n}(I)$.

(b) It holds by similar argument.

Theorem 3.21:

Let $f: V_{n}(I) \rightarrow U_{n}(I)$ be a strong n-refined neutrosophic homomorphism. Then

(a) $\operatorname{Ker}(f)$ is a strong n-refined neutrosophic subspace of $V_{n}(\mathrm{I})$.

(b) $\operatorname{Im}(f)$ is a strong n-refined neutrosophic subspace of $U_{n}(I)$.

Proof:

(a) $f$ is a module homomorphism since $V_{n}(I), U_{n}(I)$ are modules over the n-refined neutrosophic field $K_{n}(I)$, hence $\operatorname{Ker}(f)$ is a submodule of the vector space $V_{n}(I)$, thus $\operatorname{Ker}(f)$ is a strong n-refined neutrosophic subspace of $V_{n}(I)$.

(b) Holds by similar argument.

\section{Example 3.22:}

Let $R_{2}^{2}(I)=\left\{x_{0}+x_{1} I_{1}+x_{2} I_{2} ; x_{0}, x_{1}, x_{2} \in R^{2}\right\}, R_{2}^{3}(I)=\left\{y_{0}+y_{1} I_{1}+y_{2} I_{2} ; y_{0}, y_{1}, y_{2} \in R^{3}\right\}$ be two weak 2refined neutrosophic vector space over the field R. Consider $f: R_{2}^{2}(I) \rightarrow R_{2}^{3}(I)$, where

$f\left[(a, b)+(m, n) I_{1}+(k, s) I_{2}\right]=(a, 0,0)+(m, 0,0) I_{1}+(k, 0,0) I_{2}, f$ is a weak 2-refined neutrosophic homomorphism over the field $\mathrm{R}$.

$\operatorname{Ker}(f)=\left\{(0, b)+(0, n) I_{1}+(0, s) I_{2} ; b, n, s \in R\right\}$

$\operatorname{Im}(f)=\left\{(a, 0,0)+(m, 0,0) I_{1}+(k, 0,0) I_{2} ; a, m, k \in R\right\}$.

Example 3.23:

Let $W_{2}(I)=<(0,0,1) I_{1}>=\left\{q .(0,0, a) I_{1} ; a \in R, q \in R_{2}(I)\right\}, U_{2}(I)=<(0,1,0) I_{1}>=\left\{q .(0, a, 0) I_{1} ; a \in R ; q \in\right.$ $\left.R_{2}(I)\right\}$ be two strong 2-refined neutrosophic subspaces of the strong 2-refined neutrosophic vector space $R_{2}^{3}(I)$ over 2-refined neutrosophic field $R_{2}(\mathrm{I})$. Define $f: W_{2}(I) \rightarrow U_{2}(I) ; f\left[q(0,0, a) I_{1}\right]=q(0, a, 0) I_{1} ; q \in R_{2}(I)$.

$f$ is a strong 2-refinedneutrosophic homomorphism:

Let $A=q_{1}(0,0, a) I_{1}, B=q_{2}(0,0, b) I_{1} \in W_{2}(I) ; q_{1}, q_{2} \in R_{2}(I)$, we have 


$$
A+B=\left(q_{1}+q_{2}\right)(0,0, a+b) I_{1}, f(A+B)=\left(q_{1}+q_{2}\right) \cdot(0, a+b, 0) I_{1}=f(A)+f(B) .
$$

Let $m=c+d I_{1}+e I_{2} \in R_{2}(I)$ be a 2-refined neutrosophic scalar, we have

$m \cdot A=c \cdot q_{1}(0,0, a) I_{1}+d \cdot q_{1}(0,0, a) I_{1} I_{1}+e \cdot q_{1}(0,0, a) I_{2} I_{1}=q_{1}(0,0, c . a+d . a+e . a) I_{1}$,

$f(m . A)=q_{1}(0, c \cdot a+d \cdot a+e \cdot a, 0) I_{1}=m \cdot f(A)$, hence $f$ is a strong 2-refined neutrosophic homomorphism.

$\operatorname{Ker}(f)=(0,0,0)+(0,0,0) I_{1}+(0,0,0) I_{2}$.

$\operatorname{Im}(f)=U_{2}(I)$

Remark 3.24:

A union of two n-refined neutrosophic vector spaces $V_{n}(I)$ and $W_{n}(I)$ is not supposed to be an n-refined neutrosophic vector space, since the addition operation can not be defined. For example consider $V=R^{3}, W=R^{2}, n=2$.

\section{Conclusion}

In this paper we have introducedthe concept of weak/strong n-refined neutrosophic vector space. Also, some related concepts such as weak/strong n-refined neutrosophic subspace, weak/strong n-refined neutrosophic homomorphism have been presented and studied.

\section{Future research}

Authors hope that some corresponding notions will be studied in future such as weak/strong n-refined neutrosophic basis, and AH-subspaces.

Funding:: This research received no external funding

Conflicts of Interest:The authors declare no conflict of interest

\section{References}

[1]Abobala, M., "On Some Special Substructures of Neutrosophic Rings and Their Properties", International Journal of Neutrosophic Science", Vol 4, pp.72-81, 2020.

[2]Abobala, M., "On Some Special Substructures of Refined Neutrosophic Rings", International Journal of Neutrosophic Science, Vol 5, pp.59-66, 2020.

[3]Abobala, M,. "Classical Homomorphisms Between Refined Neutrosophic Rings and Neutrosophic Rings", International Journal of Neutrosophic Science, Vol 5, pp.72-75, 2020.

[4]Adeleke, E.O., Agboola, A.A.A., and Smarandache, F., "Refined Neutrosophic Rings I", International Journal of Neutrosophic Science, Vol 2, pp.77-81, 2020.

[5]Agboola, A.A.A,.and Akinleye, S.A., "Neutrosophic Vector Spaces", Neutrosophic Sets and Systems, Vol 4,pp 9$17,2014$.

[6]Agboola, A.A.A., Akwu, A.D., and Oyebo, Y.T., "Neutrosophic Groups and Subgroups", International .J .Math.Combin, Vol 3, pp.1-9, 2012.

[7]Agboola, A.A.A., Akinola, A.D., and Oyebola, O.Y.," NeutrosophicRings I ", International J.Mathcombin, Vol 4,pp.1-14, 2011. 
[8]Kandasamy, V.W.B., andSmarandache, F., "Some Neutrosophic Algebraic Structures and Neutrosophic NAlgebraic Structures", Hexis, Phonex, Arizona 2006.

[9]Olgan, N., and Khatib, A., "Neutrosophic Modules", Journal of Biostatistic and Biometric Application", Vol 3, 2018 .

[10]Smarandache, F., "Symbolic Neutrosophic Theory", EuropaNovaasbl, Bruxelles, 2015.

[11]Smarandache, F., n-Valued Refined Neutrosophic Logic and Its Applications in Physics, Progress in Physics, pp.143-146, Vol. 4, 2013.

[12]Smarandache, F., and Abobala, M., "n-RefinedNeutrosophic Rings", International Journal of Neutrosophic Science, Vol. 5, pp.83-90, 2020. 